\title{
Design and Optimization of the CFRP Mirror Components
}

\author{
Lei WEI ${ }^{1,2}$, Lei $\mathrm{ZHANG}^{2,3^{*}}$, and Xiaoxue $\mathrm{GONG}^{1,2}$ \\ ${ }^{1}$ University of Chinese Academy of Sciences, Beijing, 100039, China \\ ${ }^{2}$ Changchun Institute of Optics, Fine Mechanics and Physics, Chinese Academy of Sciences, Changchun, 130033, China \\ ${ }^{3}$ Chang Guang Satellite Technology LTD.Co, Changchun, 130000, China \\ *Corresponding author: Lei ZHANGＩE-mail: 18686344285@163.com
}

\begin{abstract}
As carbon fiber reinforced polymer (CFRP) material has been developed and demonstrated as an effective material in lightweight telescope reflector manufacturing recently, the authors of this article have extended to apply this material on the lightweight space camera mirror design and fabrication. By CFRP composite laminate design and optimization using finite element method (FEM) analysis, a spherical mirror with $\varphi 316 \mathrm{~mm}$ diameter whose core cell reinforcement is an isogrid configuration is fabricated. Compared with traditional ways of applying ultra-low-expansion glass (ULE) on the CFRP mirror surface, the method of nickel electroplating on the surface effectively reduces the processing cost and difficulty of the CFRP mirror. Through the FEM analysis, the first order resonance frequency of the CFRP mirror components reaches up to $652.3 \mathrm{~Hz}$. Under gravity affection coupling with $+5^{\circ} \mathrm{C}$ temperature rising, the mirror surface shape root-mean-square values (RMS) at the optical axis horizontal state is $5.74 \mathrm{~nm}$, which meets mechanical and optical requirements of the mirror components on space camera.
\end{abstract}

Keywords: Pure-CFRP mirror; lay-up design; bipod; integrated optimization

Citation: Lei WEI, Lei ZHANG, and Xiaoxue GONG, "Design and Optimization of the CFRP Mirror Components," Photonic Sensors, 2017, 7(3): 270-277.

\section{Introduction}

With the development of space technology, high resolution, wide field of view, and ultra lightweight are heavily required for space camera nowadays. But the mass of the primary mirror used for the space camera is increased as a sacrifice to meet the requirement. Therefore, the lightweight research on primary mirror with high optical performance has become an important issue worldwide [1].

Carbon fiber composite materials (CFRP) have the characteristics of high stiffness, low thermal expansion coefficient, and changeable material properties, which make it a possible excellent material for the space camera reflector fabrication. Due to the development of the non-contact ion beam optical fabrication method, CFRP mirror processing is no longer a challenge. Researchers from Europe and the United States have already studied on the lightweight of the CFRP mirror for many years [2-9]. Composite Optics, lnc. (COI) designed and manufactured a 2-m caliber CFRP primary mirror which works at a far-infrared wave band. Chen et al. proposed the process method using space-flightqualified graphite-fiber-reinforced cyanate ester composite materials to print mirror surface through at the mould surface and made a tremendous breakthrough in achieving the replicas with areal

Received: 2 October 2016/ Revised: 3 March 2017

(C) The Author(s) 2017. This article is published with open access at Springerlink.com

DOI: $10.1007 / \mathrm{s} 13320-017-0388-2$

Article type: Regular 
density $2 \mathrm{~kg} / \mathrm{m}$ at $42-\mathrm{cm}$ aperture by vacuum drying and ion milling [10]. Romeo et al. developed an ULTRA telescope with a 1-m caliber primary mirror [11-13].

Researches on CFRP reflector in China started relatively later than several other countries, but they are making a rapid progress. There have been several related institutions working on the CFRP reflector for several decades. For example, Peng et al. from The Shanghai Institute of Technical Physics of the Chinese Academy of Sciences have studied in mirror made of carbon fiber honeycomb sandwich panel [14]. Zhao et al. from Xi'an Institute of Optics and Precision Mechanics applied actuators to change the curvature of the mirror surface and produced the CFRP surface-changeable reflector [15].

The CFRP reflect mirror mainly adopts two structure forms now. One form applies a sandwich structure with carbon fiber material as the top and bottom panel and honeycomb material as the intermediate support $[14,16,17]$. The other form uses all carbon fiber not only as the top and bottom but also as the intermediate support [11-13, 18]. Compared with the 1 th form, the 2 nd form has better thermal suitability and higher stiffness. Therefore, this structure form satisfies space application demands better. However, great limit on the application of pure carbon fiber mirror appears because how to smooth the rough CFRP material surface is still a challenge worldwide. Due to the limitation of current technology, it's impossible to process the CFRP mirror surface at a precise optical standard. A traditional solution to this problem is to cover ultra-low-expansion glass (ULE) chip on the surface of the CFRP mirror body, but the processing of the ULE chip is difficult and costly. This article comes up with a new solution to this problemelectroplating nickel on the surface of CFRP mirror body instead of covering the ULE chip, which is also a useful way to solve the problem. Besides, an optimal design is also given in this paper.

\section{Optimized design of the reflector}

The basic requirements for optical material are low thermal coefficient of expansion, isotropic property, and high specific stiffness. However, carbon fiber represents a complicated anisotropic property, leading to variation in the elastic modulus and thermal coefficient of expansion as ply-orientation changes. In order to apply carbon fiber in optical mirrors, coupling the ULE mirror surface on honeycomb sandwich structure mirror substrate is proposed as a common method [14]. The CFRP mirror manufactured in this way can be described in Fig. 1. However, this paper adopts a different pure carbon fiber grid support reinforcement structure form instead of honeycomb sandwich structure to manufacture the CFRP mirror. The preparation processes of pure carbon fiber mirror which also take the optical material demands into account are shown in Fig. 2. The reflect mirror studied is a $\varphi 316-\mathrm{mm}$ caliber spherical CFRP mirror with $1580-\mathrm{mm}$ spherical radius.

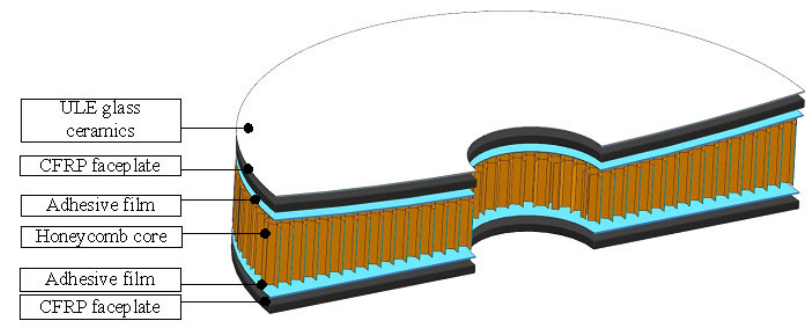

Fig. 1 Component of the CFRP reflector.

\subsection{Mirror design}

To meet with the high-specific-stiffness, isotropic, and low-thermal-expansion-coefficient characteristics of optical material, the plies used to form CFRP mirror surface should be specially laid up with high modulus fiber to ensure the quasic-isotropic property, and the resin which is used to adhere plies should maintain low thermal coefficient of expansion. Besides, low shrinkage rate, low solidified rate, and process stability and affinity to carbon fiber should also be taken into consideration for the resin. Above all, taking 
existing resources into account, this paper selects M40 fiber and epoxy resin as the CFRP mirror processing material.

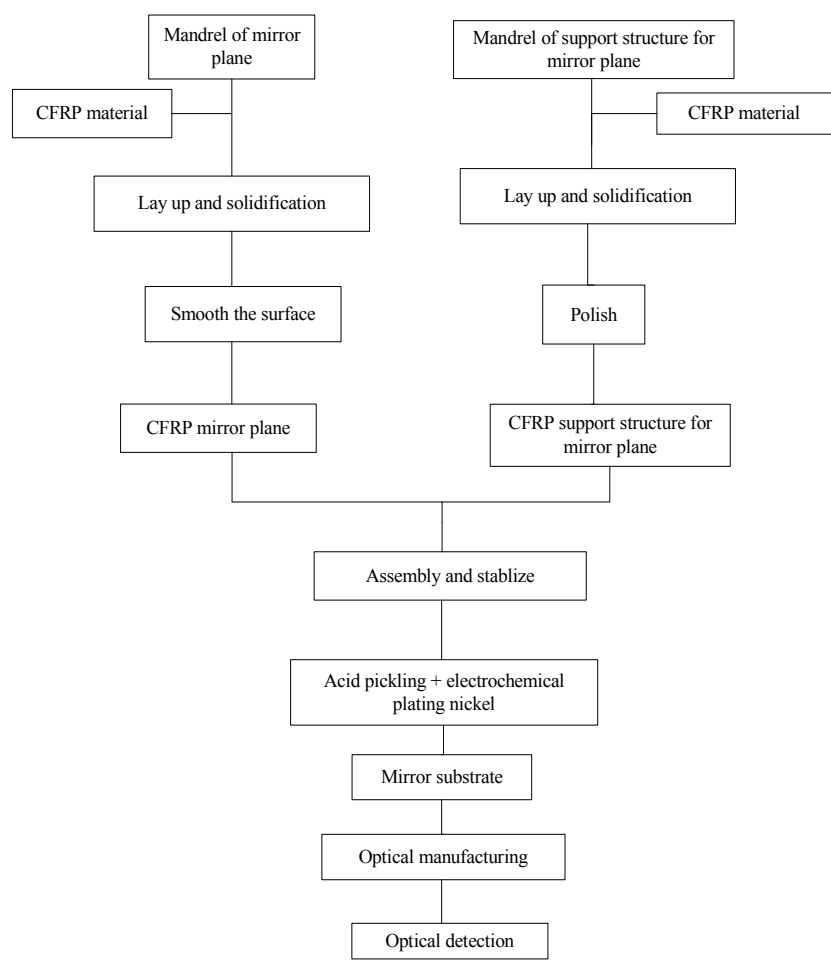

Fig. 2 Preparation processes of the CFRP reflector.

\subsubsection{Lay-up design}

The lay-up design of the mirror surface part is the key work of the whole mirror design. The quality of lay-up determines the quality of the mirror. A lay-up usually consists of several plies. The ply-orientations, the adhesive order of plies with different orientations, proportion of each orientation, and the whole ply number affect the quality of lay-up. The theoretical formulas which are always used to design the plies are described as follows:

Longitudinal tensile elastic modulus:

$$
E_{1}=E_{t} V+E_{m}\left(1-V_{t}\right)
$$

lateral tensile elastic modulus:

$$
E_{2}=\frac{E_{f} E_{m}\left(V_{f}+\eta_{2} V_{m}\right)}{E_{m} V_{f}+E_{f} V_{m} \eta_{2}}
$$

longitudinal Poisson's ratio:

$$
v_{1}=v_{f} V_{f}+V_{m}\left(1-V_{f}\right)
$$

lateral Poisson's ratio:

$$
v_{2}=v_{1} \frac{E_{1}}{E_{2}}
$$

shear modulus:

$$
G_{12}=\frac{G_{f} G_{m}\left(V_{f}+\eta_{2} V_{m}\right)}{G_{m} V_{f}+G_{f} V_{m} \eta_{2}}
$$

where $E_{f}$ and $E_{m}$ are the elastic moduluses of carbon fiber and resin, respectively, $v_{f}$ and $v_{m}$ are the corresponding Poisson's ratios, $G_{f}$ and $G_{m}$ are the corresponding shear elastic moduluses, $V_{f}$ is the fiber volume percentage, $V_{m}=1-V_{f}$ is the resin volume percentage, and $\eta_{2}$ and $\eta_{12}$ are given by experiments and equal to 0.97 for plies in this paper.

Lay-up of mirror surface part generally has the following principles:

(1) Plies should be symmetrical with the mid of the mirror surface part to prevent warping.

(2) Proportion of each ply-orientation should be no less than $6 \%-10 \%$.

(3) Ply-orientation should not be too complicated. Orientations at $45^{\circ}$ and $60^{\circ}$ are mostly chosen to ensure quasic-isotropic property of the surface part.

According to Chen et al. [19], the volume of a fiber/resin ratio should be $67 \%$ with 24 plies. Each plies is $0.1-\mathrm{mm}$ thick.

Above all, the author works on the optimization research of the ply-orientation design to find the minimal root-mean-square values (RMS) along the optical axis of mirror surface under the affection of gravity. Hypermesh is applied to the FEM analysis. Due to the principle of permutation and combination, total 15 plies orientations are selected to conduct the analysis. The analysis results are given in Table 1.

Due to the results given in Table $1,\left[0^{\circ} / 60^{\circ} /-60^{\circ}\right]$ section performs the minimum RMS and is chosen to manufacture the mirror surface. Microstructure of this section is described in Fig. 3, and the surface deformation is described in Fig.4. 
Table 1 Mirror surface deformation under the different ply-orientations combined.

\begin{tabular}{ccc}
\hline Ply fiber orientation & PV $(\mathrm{nm})$ & RMS (nm) \\
\hline$\left[0^{\circ} / 60^{\circ} /-60^{\circ}\right]_{8}$ & 6.6 & 372 \\
{$\left[0^{\circ} /-60^{\circ} / 60^{\circ}\right]_{8}$} & 6.59 & 372 \\
{$\left[60^{\circ} / 0^{\circ}-60^{\circ}\right]_{8}$} & 10.71 & 349 \\
{$\left[60^{\circ}-60^{\circ} / 0^{\circ}\right]_{8}$} & 16.4 & 352 \\
{$\left[-60^{\circ} / 60^{\circ} / 0^{\circ}\right]_{8}$} & 16.4 & 352 \\
{$\left[-60^{\circ} / 0^{\circ} / 60^{\circ}\right]_{8}$} & 7.91 & 356 \\
{$\left[-45^{\circ} / 0^{\circ} / 45^{\circ} / 90^{\circ}\right]_{6}$} & 6.88 & 377 \\
{$\left[-45^{\circ} / 90^{\circ} / 45^{\circ} / 0^{\circ}\right]_{6}$} & 16.6 & 351 \\
{$\left[0^{\circ} / 45^{\circ} / 90^{\circ} /-45^{\circ}\right]_{6}$} & 10.36 & 349 \\
{$\left[0^{\circ} /-45^{\circ} / 90^{\circ} / 45^{\circ}\right]_{6}$} & 10.36 & 349 \\
{$\left[45^{\circ} / 0^{\circ}-45^{\circ} / 90^{\circ}\right]_{6}$} & 6.9 & 377 \\
{$\left[45^{\circ} / 90^{\circ} /-45^{\circ} / 0^{\circ}\right]_{6}$} & 16.6 & 351 \\
{$\left[90^{\circ} / 45^{\circ} / 0^{\circ} /-45^{\circ}\right]_{6}$} & 10.1 & 365 \\
{$\left[90^{\circ} /-45^{\circ} / 0^{\circ} / 45^{\circ}\right]_{6}$} & 10.1 & 364 \\
\hline
\end{tabular}

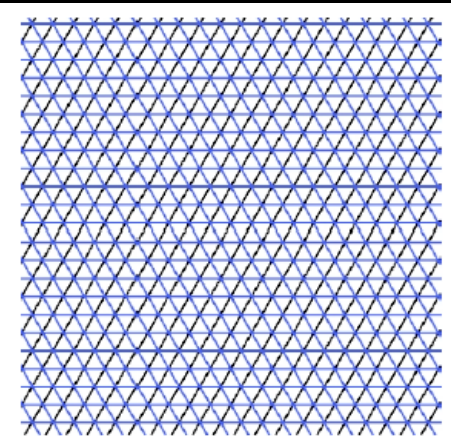

Fig. 3 Microstructure of the surface with different ply-orientations combined.

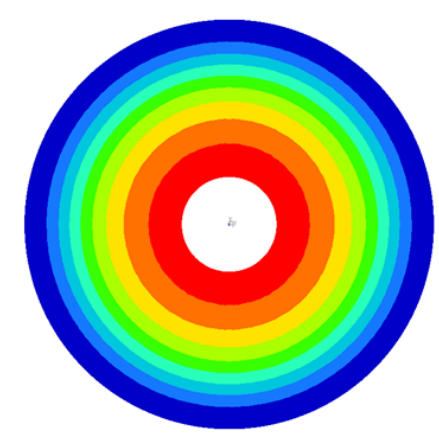

Fig. 4 Surface deformation of the reflector under loads.

\subsubsection{Mirror surface fabrication}

Carbon fiber mirror fabrication, which is also called optical surface transformation (OST), requires a pair of forming dies which are composed of convex die and concave die. In addition, the forming surface of concave die must be polished into the required shape of the mirror. With forming dies, the carbon fiber material that has been correctly laid up can mold for the mirror plane at the appropriate temperature and pressure conditions. The molding process is shown in Fig. 5.

\subsection{Fabrication of mirror body}

Different from the honeycomb structure which is adopted in the traditional reflector as mirror body, the carbon fiber grid structure mirror body has higher support stiffness and better thermal matching with the rest part. Unlike the mirror surface part $\left(\left[0^{\circ} / 60^{\circ} /-60^{\circ}\right]\right.$ style $)$, the $\left[0^{\circ} / 45^{\circ} / 90^{\circ} /-45^{\circ}\right]$ style is applied in the body fabrication because better mechanical property and thermal suitability are required for the mirror body. To ensure better thermal stability, the grid structure and its back surface are adhered together as mirror body after the dependent fabrication. To meet with the concave interface between the mirror surface part and mirror body, grid forming die should be manufactured at corresponding curvature, as shown in Fig. 6. The finished mirror body is shown in Fig. 7.
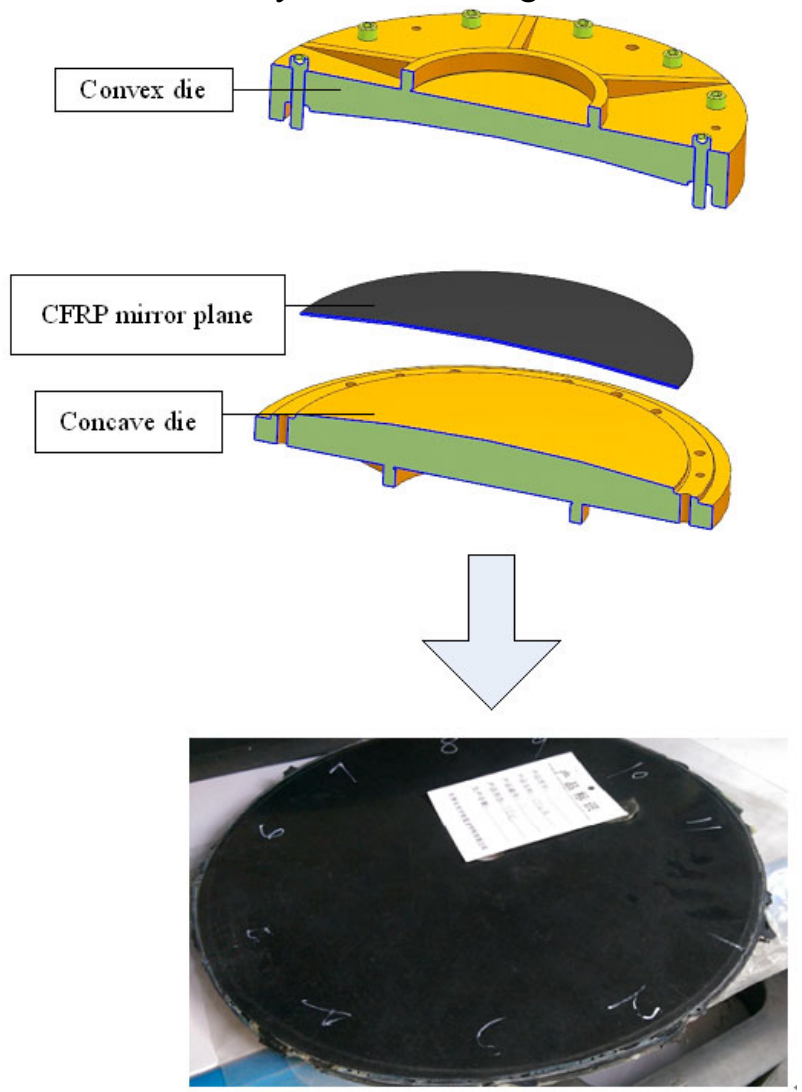

Fig. 5 Forming process of the mirror. 


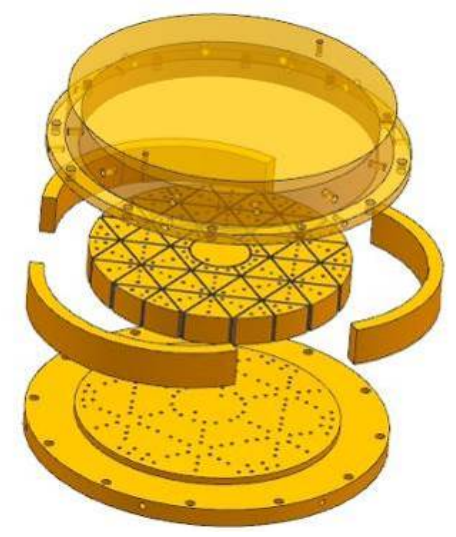

Fig. 6 Grid forming die.

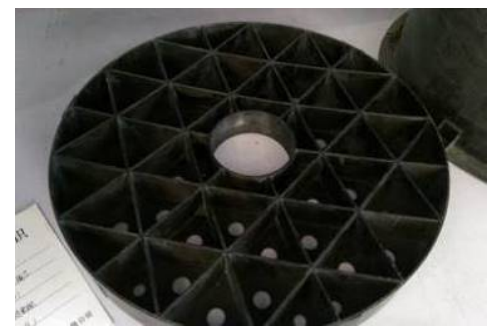

Fig. 7 Mirror body with carbon fiber grid reinforced structure.

\subsection{Carbon fiber mirror assembly and optical processing}

The carbon fiber mirror assembly processes mainly contain 3 steps as follows:

(1) Splice the mirror surface part and mirror body with epoxy resin and assembly tooling.

(2) Expose the conductive carbon fiber mirror surface by acid pickling of the epoxy resin which is left on the mirror surface during Step 1.

(3) Electroplate $0.2 \mathrm{~mm}$ thick nickel on the mirror surface.

Now that a carbon fiber mirror substrate has been manufactured, optical processing is on the next to ensure a precise mirror face. Single diamond turning of the nickel layer with ultraprecise diamond lathe is applied in mirror surface rough finishing, this method is time-saving, and a mirror surface wavefront accuracy of $0.2 \lambda(\lambda=632 \mathrm{~nm})$ can be achieved after this process. At last ion milling is applied during mirror surface fine finishing, and this non-contact finishing method can guarantee the mirror surface unstressed during the whole fine finishing process.

\section{Support design of the carbon fiber mirror}

In order to meet with the primary mirror surface deformation requirement under gravity affection coupling with $+5^{\circ} \mathrm{C}$ temperature rise at optical axis horizontal state and take mirror stiffness requirement also into consideration, 3-point peripheral bipod support as shown in Fig. 8 is adopted to support the CFRP reflector. The detail size parameters of the bipod flexure hinge are shown in Fig. 9.

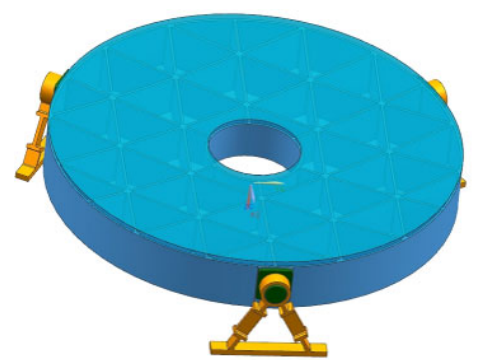

Fig. 8 Three-point peripheral bipod support around the mirror.

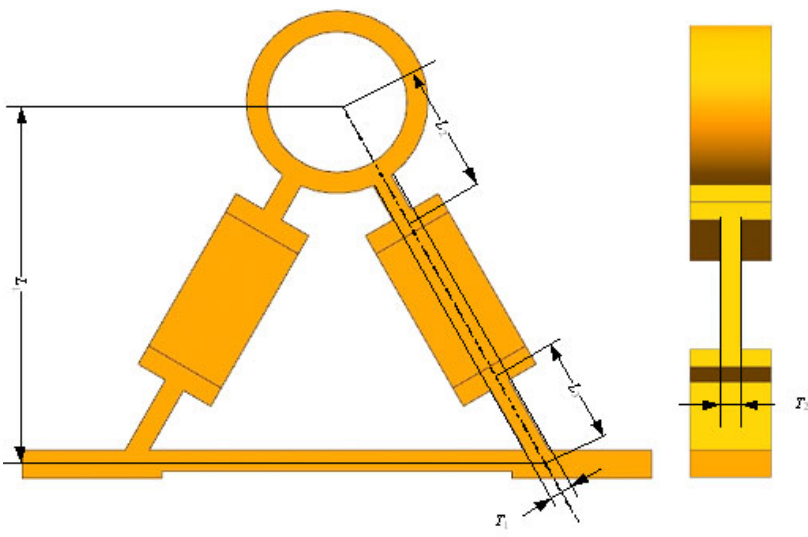

Fig. 9 Each parameter of the flexure hinge.

Set the value of $L_{1}$ as limit, and $L_{2}, L_{3}, T_{1}$, and $T_{2}$ as optimization variables, to seek the minimal RMS value under gravity affection coupling with $+5^{\circ} \mathrm{C}$ temperature rise at the optical axis horizontal state. Several programs contained of Partran, Nastran, UG, Sigfitare are integrated to complete the optimization work. The optimized model goes as follows:

(1) Optimization target

Achieve the minimal RMS value (at axis horizontal state, considering gravity affection coupling with $+5^{\circ} \mathrm{C}$ temperature rising as well). 
(2) Optimization variables

The $L_{2}, L_{3}, T_{1}$, and $T_{2}$ sizes of flexible hinge constraints:

$L_{1}=51 \mathrm{~mm}$

$8 \mathrm{~mm} \leq L_{2} \leq 30 \mathrm{~mm} ;$

$8 \mathrm{~mm} \leq L_{3} \leq 30 \mathrm{~mm} ;$

$1.5 \mathrm{~mm} \leq T_{1} \leq 5 \mathrm{~mm}$;

$1.5 \mathrm{~mm} \leq T_{2} \leq 5 \mathrm{~mm}$.

After 78 iterations, the optimization work is done, and the model converges. Results are shown in Table 2, and Fig. 10 shows the RMS value in each iteration.

Table 2 Optimized size parameters of the bipod hinge type and the surface RMS values.

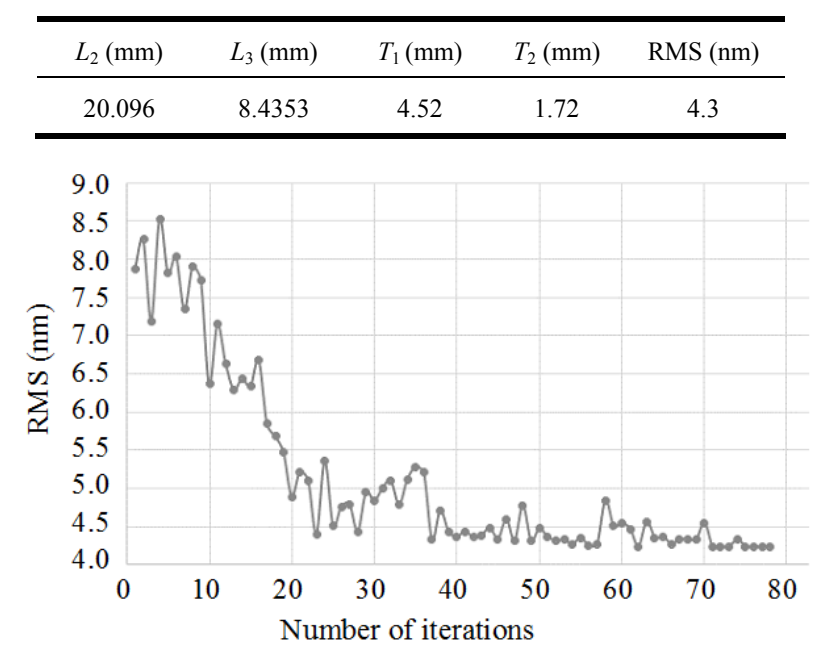

Fig. 10 Iteration history of mirror surface shape.

According to the optimal results above and processing craft, the flexible supporting structure is given in Fig. 11.

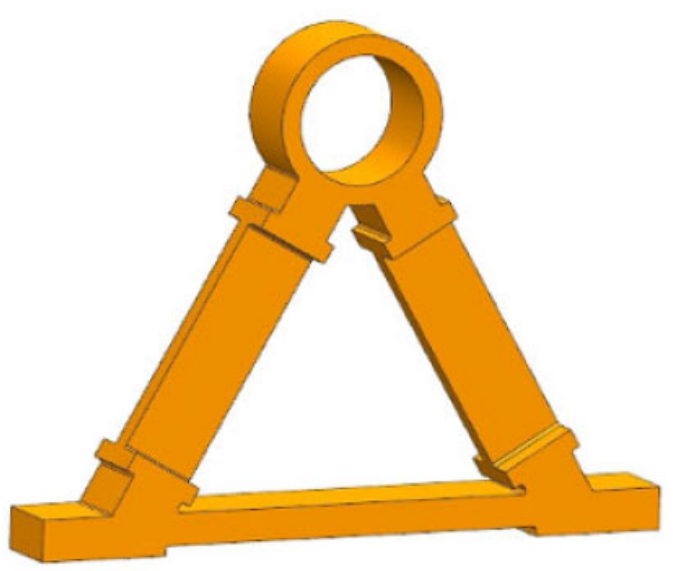

Fig. 11 Optimized structure of the bipod support.

\section{Analysis and validation}

Apply the FEM analysis for CFRP mirror, the FEM meshes are shown in Fig. 12, and the analysis results are shown in Table 3.

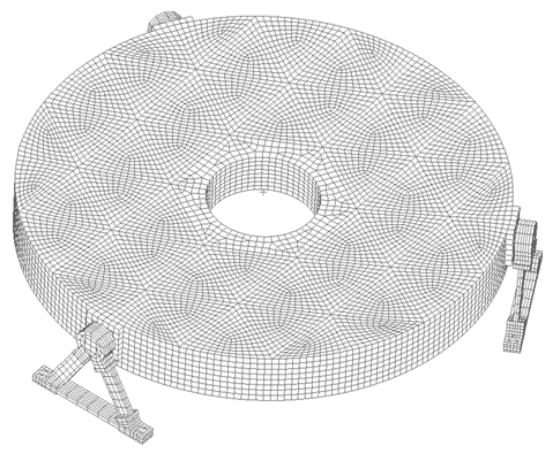

Fig. 12 Finite model of the CFRP mirror.

Table 3 FEM analysis results.

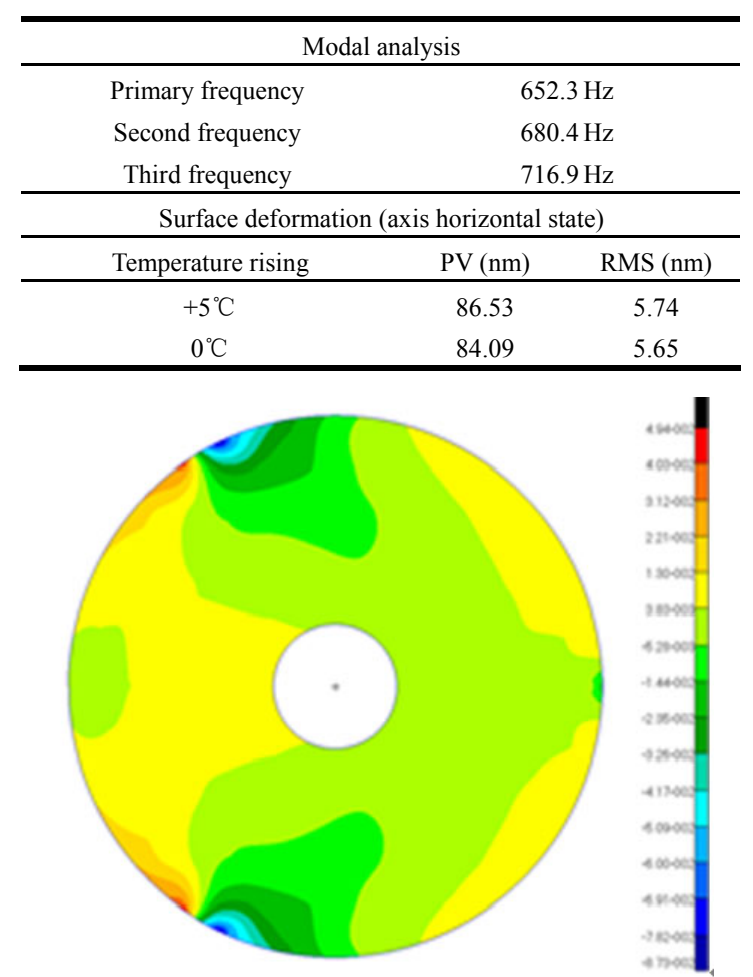

Fig. 13 Mirror surface deformation nephogram under gravity affection coupling with $+5^{\circ} \mathrm{C}$ temperature rise at optical axis horizontal state.

Considering gravity affection coupling with $+5{ }^{\circ} \mathrm{C}$ temperature rising, the mirror surface deformation on the detecting direction (axis horizontal state) is shown in Fig. 13. Based on the analysis results above, the RMS value of designed CFRP mirror components is $5.74 \mathrm{~nm}$, less than $\lambda / 50$ 
$(\lambda=632 \mathrm{~nm})$, which satisfies the space mirror demand at visible wavelengths. The primary frequency $(652.3 \mathrm{~Hz})$ of designed CFRP mirror components is far higher than $100 \mathrm{~Hz}$, which satisfies the requirement during rocket launch.

\section{Conclusions}

The authors have designed a CFRP mirror and its supports for space camera, and the CFRP mirror substrate which is assembled by carbon fiber mirror surface part and carbon fiber mirror body has been fabricated. The carbon fiber mirror surface part applies the FEM method in the ply-orientation optimal design and finds the best result at $\left[0^{\circ} / 60^{\circ} /-60^{\circ}\right]$ style. The mirror body applies carbon fiber grid reinforced structure instead of honeycomb. Three-point peripheral bipod flexure hinge support is chosen to support the mirror. An integrated optimization analysis has revealed the best structure parameters of bipod support. Through the FEM analysis of the whole components, the primary frequency of the designed CFRP mirror components reaches up to $652.3 \mathrm{~Hz}$, and the RMS value of the mirror surface is $5.74 \mathrm{~nm}$ under gravity affection coupling with $+5^{\circ} \mathrm{C}$ temperature rise at optical axis horizontal state, which meet with the mechanical and optical requirements of space camera. Researches in this paper will offer great help to future applications of CFRP mirror on space camera.

\section{Acknowledgement}

This work was supported by the National Natural Science Foundation of China (NSFC) (No. 41501383).

Open Access This article is distributed under the terms of the Creative Commons Attribution 4.0 International License (http://creativecommons.org/ licenses/by/4.0/), which permits unrestricted use, distribution, and reproduction in any medium, provided you give appropriate credit to the original author(s) and the source, provide a link to the Creative Commons license, and indicate if changes were made.

\section{References}

[1] B. He and Z. L Li, "Design of the strip SiC mirror supporting structure and lightweight," Optical Technique, 2008, 4(34): 593-596.

[2] P. B. Willis and D. R. Coulter, "Durability and reliability of lightweight composite mirrors for space optical systems," SPIE, 1993, 1993: 127-136.

[3] M. E. L. Jungwirth, C. C. Wilcox, D. V. Wick, J. Robichaud, R. C. Romeo, R. N. Martin, et al., "Large-aperture active optical carbon fiber reinforced polymer mirror," SPIE, 2013, 8725: 1-11.

[4] C. C. Wilcox, M. E. L. Jungwirth, D. V. Wick, R. C. Romeo, and R. N. Martin, "Closed-loop performance of an actuated deformable carbon fiber reinforced polymer mirror," SPIE, 2012, 8373: .

[5] S. Kendrew and P. Doel, "Development of a carbon fibler composite active mirror: design and testing," Optical Engineering, 2006, 45(3): 535-545.

[6] P. Doel, S. Kendrew, D. Brooks, C. Dorn, C. Yates, R. D. Martin, et al., "Development of an active carbon fiber composite mirror," SPIE, 2004, 5490: $1526-1533$.

[7] M. E. L. Jungwirth, M. Baker, D. V. Wick, C. C. Wilcox, R. C. Romeo, R. D. Martin, et al., "Actuation for carbon fiber reinforced polymer active optical mirrors," in Proc. of 2012 IEEE Aerospace Conference, Big Sky, Montana, 2012, pp. 1-9.

[8] Y. Arao, J. Koyanagi, S. Utsunomiya, S. I. Takeda, and H. Kawada, "Analysis of time-dependent deformation of a CFRP mirror under hot and humid conditions," Mechanics of Time-Dependent Materials, 2009, 13(2): 183-197.

[9] S. Tanaka, T. Ikeda, and A. Senba, "Sensitivity analysis of thermal deformation of CFRP laminate reflector due to fiber orientation error," Journal of Mechanical Science \& Technology, 2016, 30(10): 4423-4426.

[10] P. C. Chen, T. T. Saha, A. M. Smith, and R. Romeo, "Progress in very lightweight optics using graphite fiber composite materials," Optical Engineering, 1998, 37(2): 666-676.

[11] R. C. Romeo and R. D. Martin, "Final assembly of the ULTRA 1-m carbon fiber optical telescope," SPIE, 2007, 6665: 1-10.

[12] R. C. Romeo and R. D. Martin, "Progress in $1 \mathrm{~m}$-class lightweight CFRP composite mirrors for the ULTRA telescope," SPIE, 2006, 6273: 1-12.

[13] R. D. Martin and R. C. Romeo, "Lightweight optical telescope structures fabricated from CFRP composites," SPIE, 2007, 6665: 1-7.

[14] W. C. Peng, S. L. Sun, G. L. Chen, L. W. Sun, and P. J. Zhang, "Carbon fiber reinforced composites for lightweight optical mirrors," Optical Technique, 
2006, 8 (32): 138-142.

[15] H. Zhao, X. W. Fan, Z. H. Pang, G. T. Ren, W. Wang, Y. J. Xie, et al., "Carbon-fiber-reinforced polymer variable-curvature mirror used for optical zoom imaging: prototype design and experimental demonstration," Optical Engineering, 2015, 54(2): 025103 .

[16] D. E. Krumweide, G. D. Wonacott, P. M. Woida, R. Q. Woida, and W. Shih, "Carbon-carbon mirrors for exoatmospheric and space applications," SPIE, 2007, 6666: $1-8$.

[17] S. Utsunomiya, T. Kamiya, and R. Shimizu, “CFRP composite mirrors for space telescopes and their micro-dimensional stability," SPIE, 2010, 7739: 1-7.

[18] G. J. Barber, A. Braem, N. H. Brook, W. Cameron, C. D'Ambrosio, N. Harnew, et al., "Development of lightweight carbon-fiber mirrors for the RICH 1 detector of LHCb," Nuclear Instruments and Methods in Physics Research, 2008, 593(3): 624-637.

[19] P. C. Chen, C. W. Bowers, D. A. Content, M. Marzouk, and R. C. Romeo, "Advances in very lightweight composite mirror technology," Optical Engineering, 2000, 39(9): 2320-2329. 